\title{
An Analysis of Ultrastructure of the Hair of Children Treated With Valproic Acid
}

\author{
Kursat Bora Carman ${ }^{\mathrm{a}, \mathrm{d}}$, Serap Sirvanci ${ }^{\mathrm{b}}$, Duygu Gursoy ${ }^{\mathrm{b}}$, Esra Carman ${ }^{\mathrm{c}}$, \\ Coskun Yarar ${ }^{\mathrm{a}}$
}

\begin{abstract}
Background: Valproic acid (VPA) is one of the most widely used antiepileptic drugs. Hair loss, color changes, thinning, and curliness have been reported in patients treated with VPA. In the present study, we aimed to analyze the ultrastructure of the hair of children treated with VPA via electron microscope.
\end{abstract}

Methods: This cross-sectional study was performed with 12 epileptic children who had been treated with VPA for at least 12 months. The hair samples of children were examined via electron microscopy.

Results: Scanning electron microscopy of hairs in patients demonstrated normal ultrastructural appearance of the hair surface and cuticular pattern.

Conclusion: The present study is retrospective. The drug-induced changes might be temporary. Therefore, prospective research projects involving larger groups might be helpful.

Keywords: Valproic acid; Hair; Analysis; Electron microscopy

\section{Introduction}

Valproic acid (VPA) is an antiepileptic drug widely used in the treatment of epilepsy as well as bipolar disorders [1]. Gastrointestinal complaints, weight gain, liver dysfunction, and thrombocytopenia are well-defined side effects of VPA [2]. It has been reported that VPA has several effects on the hair growth, including hair loss, thinning of the hair, and hair color changes

Manuscript accepted for publication November 01, 2016

aDepartment of Pediatric Neurology, Eskisehir Osmangazi University Hospital, Eskisehir, Turkey

bepartment of Histology and Embryology, Faculty of Medicine, Marmara University, Istanbul, Turkey

'Department of Dermatology, Eskisehir State Hospital, Eskisehir, Turkey

${ }^{\mathrm{d} C o r r e s p o n d i n g ~ A u t h o r: ~ K u r s a t ~ B o r a ~ C a r m a n, ~ B u y u k d e r e ~ M a h, ~ B i r k o n a k ~ S i-~}$ tesi, B Blok No.22, Eskisehir, Turkey. Email: kbcarman@gmail.com

doi: https://doi.org/10.14740/ijcp257w
[3-6]. The prevalence of VPA-induced hair texture changes was reported to be $11 \%$ in a study done in adults [7]. The effects of VPA on hair occur usually after 3 - 6 months of the treatment [3]. Although different kinds of hair changes have been reported, the ultrastructure of the hair of children treated with VPA has not been adequately explored.

In the present study, we aimed to analyze the ultrastructure of the hair of children treated with VPA via electron microscope.

\section{Materials and Methods}

The present study was performed between November 2015 and February 2016 at Department of Pediatric Neurology. Epileptic children who had been treated with VPA for at least 12 months were taken as research group. All children had had generalized convulsions and their brain magnetic resonance imaging was normal. All children reported that they were suffering from hair loss with varying degrees severity that had occurred after the initiation of VPA therapy. The physical and neurological examinations of all children were normal, and all had been seizurefree for at least 12 months. The blood VPA levels were measured and the daily dosage of drug was calculated in $\mathrm{mg} / \mathrm{kg} / \mathrm{day}$. To exclude the diseases affecting scalp, all children were examined by a dermatologist. Two healthy non-epileptic children were accepted as control. All hair samples were collected in follow-up visits and examined at the Electron Microscopy Research Unit of Marmara University, Faculty of Medicine.

The hair samples were mounted on metal stubs with double-sided adhesive carbon band. The samples were coated with gold in a BIO-RAD SC502 coating apparatus. Then, they were examined and photographed under a JEOL 5200 JSM scanning electron microscope (Tokyo, Japan). The surfaces and cuticular patterns of the hair shaft were investigated.

\section{Results}

The research group was composed of 12 epileptic children (nine females and three males) with a mean age of $11.08 \pm 3.26$ years (Table 1). The mean dosage and the mean blood level of VPA were measured as $28.66 \pm 6.67 \mathrm{mg} / \mathrm{kg} /$ day and $88.58 \pm$ $23.92 \mu \mathrm{g} / \mathrm{L}$, respectively. The mean duration of treatment was 
Table 1. The Features of the Research Group

\begin{tabular}{llllll}
\hline Patient no. & Gender & Age (years) & VPA dose $(\mathbf{m g} / \mathbf{k g} / \mathbf{d a y})$ & Blood VPA level $(\boldsymbol{\mu g} / \mathbf{m L})$ & Duration of treatment $(\mathbf{m o n t h s})$ \\
\hline 1 & Male & 10 & 23 & 95.1 & 12 \\
\hline 2 & Female & 8 & 27 & 84.1 & 24 \\
3 & Male & 15 & 32 & 92.8 & 32 \\
\hline 4 & Female & 17 & 28 & 102 & 18 \\
\hline 5 & Female & 7 & 20 & 52 & 30 \\
\hline 6 & Female & 11 & 26 & 76 & 13 \\
7 & Female & 10 & 34 & 92.7 & 41 \\
\hline 8 & Female & 10 & 21 & 64.3 & 15 \\
9 & Female & 10 & 42 & 132 & 22 \\
10 & Female & 7 & 23 & 54 & 26 \\
11 & Female & 15 & 36 & 112 & 18 \\
\hline 12 & Male & 13 & 32 & 106 & 26 \\
\hline
\end{tabular}

\section{$22.33 \pm 8.68$ months.}

The results of laboratory investigations, including thyroid function tests, complete blood count, serum electrolytes, serum folate, vitamin B12 levels, and ferritin were all within normal limits. The dermatological examinations of all children were all normal.

Scanning electron microscopy of hairs in both patients (Fig. 1) and control (Fig. 2) demonstrated normal ultrastructural appearance of the hair surface and cuticular pattern.

\section{Discussion}

VPA is an antiepileptic molecule widely used. Hair loss, color changes, thinning, and curliness have been reported in patients treated with VPA [3-6]. In a study, 2\% of patients were found to develop curliness of the hair [6]. Those effects occurred usually after 3 - 6 months of treatment [3]. In the present study,

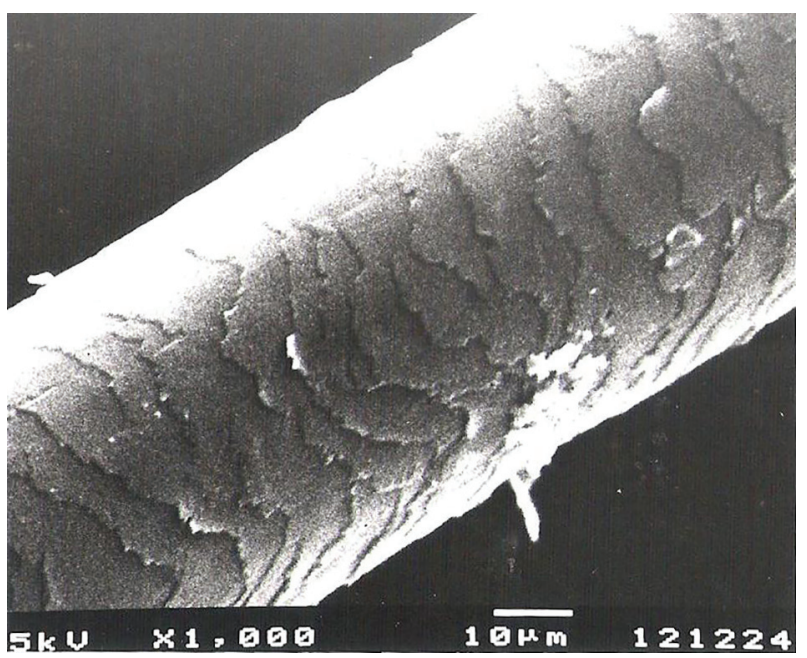

Figure 1. Normal ultrastructure of the surface and cuticular pattern of the hair shaft in a patient receiving valproic acid. we examined the hair samples of children treated for at least 12 months, and we revealed normal ultrastructure via electron microscope. VPA-induced hair texture changes may be temporary. Wilting et al [3] reported a 47-year-old female patient who suffered from curling of her hair 1 year after the initiation of VPA treatment. The curling of hair was subsided 5 years later. None of our children reported curling of the hair previously.

The mean blood level of VPA was measured as $88.58 \pm$ $23.92 \mu \mathrm{g} / \mathrm{mL}$. Gerstner et al [8] reported a 7-year-old male patient who developed changes in hair structure and color after 5 months of VPA treatment. It was reported that those changes occurred after his blood VPA level increased from 135 to 160 $\mu \mathrm{g} / \mathrm{mL}$.

The mechanism of VPA-induced hair changes is unknown. The suggested mechanism is effects of chelating of metals as well as inhibition of metallic enzymes which are essential to hair growth and keratinization [9]. One study reported changes

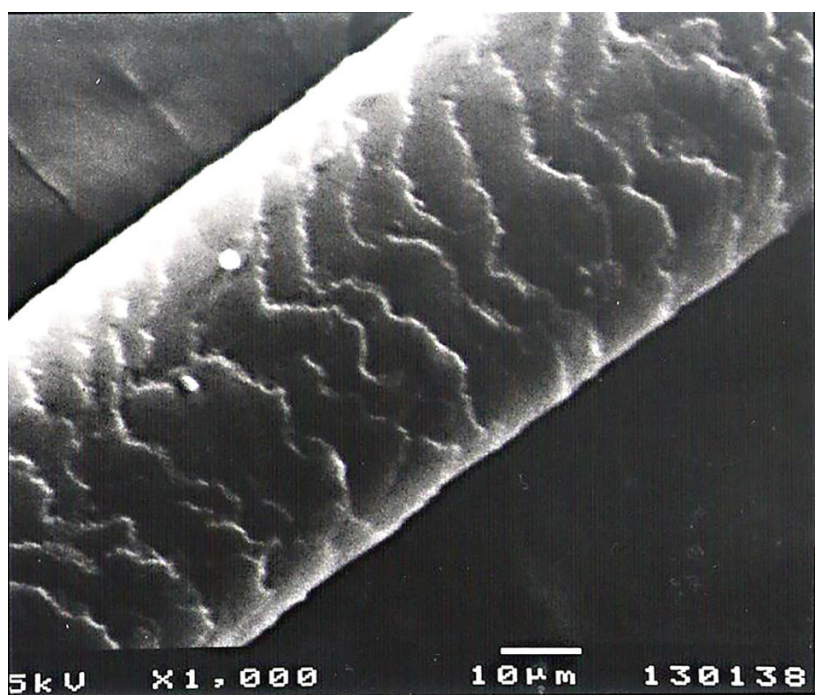

Figure 2. Control group: normal ultrastructure of the hair surface and cuticular pattern. 
in copper, zinc, and magnesium quantity in the hair of patients treated with antiepileptic drugs (carbamazepine, diphenylhydantion, phenobarbital, and VPA) as compared with health control and schizophrenic patients $[10,11]$.

Although the main topic of present research was the adverse effects of VPA on hair, Lee et al [11] reported that VPA might activate the $\mathrm{Wnt} / \beta$-catenin pathway which is important for hair follicle development. During the anagen phase of the hair cycle, activation of the $\mathrm{Wnt} / \beta$-catenin pathway is required for initiation of hair follicle formation, and stimulates the growth and differentiation of hair by maintaining expression of genes that function. In that study, it was reported that VPA did not induced hair re-growth effect when VPA was orally administered to C57BL/6 mice. However, topical application of VPA significantly promoted hair formation in murine models. The levels of $\beta$-catenin in the mice skin were specifically increased via topical VPA.

The neurological examinations of our patients were normal and all patients were primary idiopathic epileptic. The analysis of the hair of epileptic children by either light or electron microscope might provide some other benefits. Smith et al [12] investigated 322 hair samples and reported that the observed abnormalities indicated a specific diagnosis such as Menkes disease in 32\% of neurologically impaired children.

The analysis of hair can be an important diagnostic and assessment aid in medical practice and clinical evaluation of drug efficacy. The use of hair analysis has been advocated as a method for assessing therapeutic compliance to antiepileptic drug regimens. It has been well demonstrated that the major antiepileptics, phenytoin, carbamazepine, and VPA are readily recovered and quantified from hair specimens [13]. In the present study, only blood valproic level was measured, and hair samples were not investigated to analyze VPA concentration. This point might be a limitation of the present study.

In conclusion, the results of present study revealed that the ultrastructure of hair was normal in children treated with VPA. Prospective research projects involving larger groups might be helpful.

\section{Competing Interests}

All authors declare that they have no competing interests.

\section{Author Contributions}

KBC, EC and CY examined and treated the patients. SS and
DG analyzed the samples. KBC and EC wrote the manuscript. All authors read and approved the final manuscript.

\section{References}

1. Peterson GM, Naunton M. Valproate: a simple chemical with so much to offer. J Clin Pharm Ther. 2005;30(5):417421.

2. Chateauvieux S, Morceau F, Dicato M, Diederich M. Molecular and therapeutic potential and toxicity of valproic acid. J Biomed Biotechnol. 2010;2010.

3. Wilting I, van Laarhoven JH, de Koning-Verest IF, Egberts AC. Valproic acid-induced hair-texture changes in a white woman. Epilepsia. 2007;48(2):400-401.

4. Bublin JG, Thompson DF. Drug-induced hair colour changes. J Clin Pharm Ther. 1992;17(5):297-302.

5. Gupta AK. 'Perming' effects associated with chronic valproate therapy. Br J Clin Pract. 1988;42(2):75-77.

6. Jeavons PM, Clark JE, Harding GF. Valproate and curly hair. Lancet. 1977;1(8007):359.

7. Covanis A, Gupta AK, Jeavons PM. Sodium valproate: monotherapy and polytherapy. Epilepsia. 1982;23(6):693720 .

8. Gerstner T, Lipinski C, Longin E, Konig S. Valproateinduced change in hair color. J Am Acad Dermatol. 2008;58(2 Suppl):S63-64.

9. Okazaki K, Tsukida S, Morikawa F. Hair color changes during hair growth cycle in $\mathrm{C} 3 \mathrm{H}$-strain mice. Biology and Diseases of the Hair. 1976;6:489-503.

10. Suzuki T, Koizumi J, Moroji T, Shiraishi H, Hori T, Baba A, Kawai N, et al. Effects of long-term anticonvulsant therapy on copper, zinc, and magnesium in hair and serum of epileptics. Biol Psychiatry. 1992;31(6):571-581.

11. Lee SH, Yoon J, Shin SH, Zahoor M, Kim HJ, Park PJ, Park WS, et al. Valproic acid induces hair regeneration in murine model and activates alkaline phosphatase activity in human dermal papilla cells. PLoS One. 2012;7(4):e34152.

12. Smith VV, Anderson G, Malone M, Sebire NJ. Light microscopic examination of scalp hair samples as an aid in the diagnosis of paediatric disorders: retrospective review of more than 300 cases from a single centre. J Clin Pathol. 2005;58(12):1294-1298.

13. Mieczkowski T, Tsatsakis AM, Kruger M, Psillakis T. The concentration of three anti-seizure medications in hair: the effects of hair color, controlling for dose and age. BMC Clin Pharmacol. 2001;1:2. 